\title{
Data needs in child maltreatment response
}

\author{
Solving the problem begins with accurately measuring its occurrence
}

Graham V Vimpani AM, MBBS, PhD, FRACP Professor of Community and Family Health,' and and Family Health, and
Senior Clinical Advisor Child Protection and Wellbeing ${ }^{2}$

1 Paediatrics and Child Health, University of Newcastle, Newcastle, NSW.

2 NSW Kids and Families, NSW Ministry of Health, Sydney, NSW.

graham.vimpani@ hnehealth.nsw.gov.au

doi: 10.5694/mjal4.00650

(1)
and substantiation data from statutory child protection agencies to monitor child maltreatment are well known. These data are not good measures of the true prevalence of child abuse and neglect, because they are subject to changes in legislation and reporting policies and practices. ${ }^{3}$ This is nowhere more evident than in the impact of the recent change to the reporting threshold in New South Wales, changed by legislation after the Wood Inquiry in 2008, from "harm" to "significant harm". ${ }^{4}$ The number of children who were the subject of a report increased steadily from the early 2000s, reaching 114765 in the financial year 2008-09 before the NSW Government's Keep Them Safe reforms ${ }^{5}$ were introduced, but then falling to 61132 in 2010-11. ${ }^{6}$ Comparisons between and within jurisdictions over time are thus difficult, as illustrated by the fact that Researchp 162 the Australian Institute of Health and Welfare noted in its annual review of child protection in Australia that the changes meant that new data were not comparable with those from previous years. ${ }^{7}$ Use of these population data to gauge the impact of prevention and early intervention strategies - a central element of the public health approach to child protection advocated for the past 40 years ${ }^{2,3}-$ is highly problematic.

Alternative measures include the use of mortality and hospital morbidity data. ${ }^{8}$ The article by Guthridge and colleagues in this issue of the Journal, examining trends in hospital admissions for child maltreatment-related conditions in the Northern Territory, provides an example of the usefulness of the latter. ${ }^{9}$ The WHO and UNICEF have called for uniform reporting procedures for registering both fatal and non-fatal child maltreatment, arguing that health professionals are better placed than others to obtain evidence of maltreatment, and advocating for better systems of communication between health professionals and statutory child protection workers. ${ }^{10}$

The use of hospital morbidity data for surveillance of child maltreatment is not without its pitfalls, though. For a case to be coded under a definitive maltreatment code using the ICD-10-AM (International Classification of Diseases, version 10, Australian modification), ${ }_{1}^{11}$ clear clinical documentation of evidence of maltreatment is necessary. If documentation shows that an injury, for example, is queried as suspicious, but there is no documentation of further investigation being done to rule out or substantiate maltreatment, coding rules prevent the assignment of a definitive maltreatment code but allow for the case to be 
considered as possible maltreatment. The lack of clear, legible, concise and complete documentation is likely to result in underestimates of the true magnitude of maltreatment. ${ }^{7}$ Nevertheless, a study in the United States linking hospital morbidity data with child protection service data found that $12 \%$ more cases of maltreatment were identified using data from emergency departments and admissions compared with child protection service data alone. ${ }^{12}$ The value of linking data from multiple sources has also been highlighted in Western Australia, ${ }^{13}$ where data showed rises in both hospital morbidity rates and child protection notification rates over the same period.

One way in which documentation in medical records might be improved, enabling coding to achieve higher sensitivity, would be the use of agreed protocols for recording histories and the results of examinations and investigations in suspected cases of child maltreatment. A SCAN (suspected child abuse and neglect) medical protocol has recently been launched in NSW public hospitals for use by paediatric consultants and junior staff involved in assessing suspected cases of child maltreatment referred to them by other staff (emergency department, inpatient) or by the statutory agency, which has the legislative authority to request that carers present their child for a medical examination. ${ }^{14}$

Another way of improving the capture of possible cases of child maltreatment would be to broaden the coding rules to enable cases to be assigned definitive codes in suspected cases where there is undetermined intent or adverse social circumstances related to the injury. ${ }^{15}$

However, the challenges facing the development of more broadly based surveillance systems are considerable. For example, a review analysing the steps needed to develop a measurement system for inflicted brain injury concluded that the ideal system will need to link data from different sources - medical, legal and social service - and be maintained over time. ${ }^{16}$ This challenge reaffirms the growing recognition that "wicked" problems like child maltreatment inevitably require high-level strategic leadership and the good will of many to devise a collective solution.

Competing interests: No relevant disclosures.

Provenance: Commissioned; externally peer reviewed.

1 Butchart A. Epidemiology: the major missing element in the global response to child maltreatment? Am J Prev Med 2008; 34 (4 Suppl): S103-S105.

2 Gilbert R, O'Donnell M, Gonzalez-Izquierdo A, et al. Child maltreatment: variation in trends and policies in six developed countries. Lancet 2012; 379: 758-772.

3 O'Donnell M, Scott D, Stanley F. Child abuse and neglect - is it time for a public health approach? Aust N Z J Public Health 2008; 32: 325-330.

4 Wood J. Report of the Special Commission of Inquiry into Child Protection Services in NSW. Sydney: New South Wales Government, 2008.

5 New South Wales Government. Keep Them Safe: a shared approach to child wellbeing. Sydney: NSW Government, 2009.

6 NSW Department of Family and Community Services. KiDS - Corporate Information Warehouse annual data 2004-2013. Sydney: 2014.

7 Australian Institute of Health and Welfare. Child protection Australia 2011-12. Canberra: AlHW, 2013. (AlHW Cat. No. CWS 43; Child Welfare Series No. 55.)

8 McKenzie K, Scott DA. Using routinely collected hospital data for child maltreatment surveillance: issues, methods and patterns. BMC Public Health 2011; 11: 7.

9 Guthridge SL, Ryan P, Condon JR, et al. Trends in hospital admissions for conditions associated with child maltreatment, Northern Territory, 1999-2010. Med J Aust 2014; 201: 162-166.

10 Arie S. WHO takes up the issue of child abuse. BMJ 2005; 331: 129.

11 National Centre for Classification in Health. The international statistical classification of diseases and related health problems, 10th revision, Australian modification. Sydney: University of Sydney, 2008.

12 Schnitzer PG, Slusher P, van Tuinen M. Child maltreatment in Missouri: combining data for public health surveillance. Am J Prev Med 2004; 27: 379-384.

13 O'Donnell M, Nassar N, Leonard H, et al. Monitoring child abuse and neglect at a population level: patterns of hospital admissions for maltreatment and assault. Child Abuse Neglect 2010; 34: 823-832.

14 Children and Young Persons (Care and Protection) Act 1998 (NSW). http:// www.austlii.edu.au/au/legis/nsw/consol_act/caypapal998442 (accessed Jul 2014).

15 Gonzalez-Isquierdo A, Ward A, O'Donnell M, et al. Cross-country comparison of victimisation-related injury admission in children and adolescents in England and Western Australia. BMC Health Serv Res 2013; 13: 260.

16 Runyan $D$. The challenges of assessing the incidence of inflicted traumatic brain injury: a world perspective. Am J Prev Med 2008; 34 (4 Suppl): S112-S115. 